Bojana Kovačević Petrović /

UDC: $811.134 .2^{\prime 2} 255$

$811.163 .41^{\prime} 255$

DOI: https:/ / doi.org/10.18485/beoiber.2020.4.1.5

\author{
Bojana Kovačević Petrović1 \\ Nataša Popović2 \\ Universidad de Novi Sad \\ Serbia
}

\title{
CONTEXTO CULTURAL EN LA TRADUCCIÓN DE ASTÉRIX AL ESPAÑOL Y AL SERBIO3
}

\begin{abstract}
Resumen
Partiendo de las características de la traducción de tebeos en general, en este artículo hemos enfocado nuestra investigación en uno de los cómics globalmente conocidos. Considerando la traducción de los tebeos como la rama traductológica por excelencia, puesto que el traductor debe transmitir el mensaje del discurso conciso -a veces diverso y complejo y siempre relacionado con la imagen correspondiente- al otro idioma y en un espacio limitado, el artículo versa sobre la importancia del contexto cultural en ese tipo de traducción, basándose en el análisis de diez histori(et)as de Astérix, llevadas a cabo en diversos territorios: Hispania, Bélgica, Roma, Bretaña, Helvecia.... Teniendo en cuenta que todos los cuadernos de René Goscinny y Albert Uderzo están traducidos tanto al español como al serbio, hemos hecho un análisis comparativo de procedimientos, aproximaciones y soluciones que los traductores Víctor Mora y Đorđe Dimitrijević han aplicado en sus respectivas traducciones, explorando juegos verbales, particularidades lingüísticas, humor, frases hechas y expresiones coloquiales. El objetivo de esta investigación es comprobar la diferencia entre la traducción del discurso del cómic a un idioma romance (el español) y a un idioma eslavo (el serbio), así como explorar los matices contextuales, las intertextualidades y los estereotipos, a propósito de averiguar de qué manera estos elementos han sido transmitidos o perdidos durante el procedimiento de traducción. Por tanto, hemos concluido que el hecho de que una lengua es romance y otra eslava no tiene una importancia crucial y no influye el

1 bojanakp@ff.uns.ac.rs

2 natasa.popovic@ff.uns.ac.rs

3 El presente trabajo ha sido realizado dentro de los proyectos de investigación "Los aspectos de la identidad y su formación en la literatura serbia" (ON-178005) y "Lenguas y culturas en tiempo y espacio" (no 178002), financiados por el Ministerio de Educación, Ciencia y Desarrollo Tecnológico de la República de Serbia, y ha sido presentado en breve en el congreso Estudios románicos antes y ahora, llevado a cabo en la Facultad de Filosofía de la Universidad de Novi Sad, el 12 de mayo de 2019.
\end{abstract}


entendimiento del discurso, pero sí contiene los matices diferentes debido a la habilidad de los traductores.

Palabras clave: traducción de cómics, contexto cultural, Astérix en español, Astérix en serbio, humor.

\title{
CULTURAL CONTEXT IN THE TRANSLATION OF ASTÉRIX INTO SPANISH AND SERBIAN LANGUAGE
}

\begin{abstract}
Summary
Based on the characteristics of the translation of comics in general, in this article we have focused our research on one of the globally known comics. Considering the translation of comics as a translation branch par excellence, since the translator must transmit the message of a concise discourse - sometimes diverse and complex and always related to the corresponding image - into another language and in a limited space, the article is about the importance of the cultural context in this type of translation, based on the analysis of ten stories of Asterix taking place in various territories: Hispania, Belgium, Rome, Brittany, Helvecia... Considering that all the René Goscinny and Albert Uderzo's episodes have been translated into both Spanish and Serbian, we have made a comparative analysis of procedures, approaches and solutions that the translators Víctor Mora and Đorđe Dimitrijević have applied in their respective translations, exploring verbal games, linguistic particularities, humor, phrases made and colloquial expressions. The objective of this research is to verify the difference between the translation of the comic discourse to a Romance language (Spanish) and a Slavic language (Serbian), as well as to explore contextual nuances, intertextualities and stereotypes, in order to find out how these elements have been transmitted or lost during the translation procedure. Therefore, we have concluded that the fact that one language is Romance and another Slavic is not crucial and does not influence the understanding of the speech, but it does contain different nuances due to translators' abilities.
\end{abstract}

Keywords: comic translation, cultural context, Asterix in Spanish, Asterix in Serbian, humor.

\section{Reflexiones introductorias}

Por sus características particulares, el discurso de los cómics se considera un discurso híbrido, puesto que el código verbal y el aspecto visual están estrechamente relacionados. Evidentemente, su coexistencia yuxtapuesta se ha hecho más compleja a través de las décadas, por lo cual cabe resaltar que en el caso de los tebeos más logrados y exitosos ha prevalecido un discurso matizado, una intertextualidad reconocible y una interculturalidad implícita. Por un lado, los cómics varían en la abundancia de diálogos y en los registros utilizados, pero, por otro lado, el lenguaje que utilizan es casi siempre el habla coloquial, oraciones (léxica y sintácticamente) simples, onomatopeyas, juegos de palabras, frases hechas y exclamativas, elipsis, abreviaturas e interjecciones.

Es necesario hacer hincapié que, a pesar de los espacios dedicados a la historieta en los medios de comunicación o en los coloquios académicos, las investigaciones sobre la traducción de cómics siguen siendo escasas (Brandimonte 2012: 151). Sin embargo, 
para la justificación de la presente investigación se han tenido en cuenta algunas reflexiones sobre el tópico, publicadas en las últimas tres décadas. Por cierto, hasta los años 1990, la historieta ha sido excluida «del estudio de muchas disciplinas de las que participaba como la literatura, el dibujo, la pintura, el grabado, el periodismo, el cine y otras como el teatro, el diseño y la arquitectura, a pesar de la relación que guarda con todas ellas» (Rodríguez 2019: 19). Por tal circunstancia, cabe mencionar un libro recién publicado: Cómic y traducción: preliminar teórico-práctico de una disciplina de Francisco Rodríguez Rodríguez, que trata la historia y el lenguaje del cómic en el contexto sociocultural, acompañado con el análisis traductológico en el nivel morfosintáctico, léxico-semántico, pragmático-cultural, prosódico y ortotipográfico.

Puesto que la traducción de los cómics no supone exclusivamente la traducción del texto, sino también del contexto textual-visual, esas circunstancias hacen el trabajo del traductor todavía más complejo y limitado, tanto por el espacio como por las escasas palabras utilizadas. Por consiguiente, en este trabajo queremos mostrar que la traducción de una serie de cómics exige un amplio y profundo conocimiento de la cultura, tanto del idioma original como de la lengua meta. Para este estudio, se han tomado como referencia las tres técnicas de traducción más presentes en el cómic de Astérix: la traducción literal, la equivalencia y la adaptación. Buscando respuesta a la pregunta si un traductor debería adaptar su traducción a la cultura meta, sacrificando conscientemente los matices interculturales para acercar el (co)texto al público lector, o bien mantener las características originales de los personajes para perseverar su autenticidad, a continuación realizaremos un análisis paralelo de ejemplos concretos de los diez cuadernos de Astérix, publicados en su lengua original (francesa) y en dos lenguas meta (española y serbia).

\section{Cómic, tebeo, historieta}

En el territorio de habla hispana se utilizan tres palabras para designar a una serie de láminas o un cuaderno de dibujos sucesivos vinculados con un tema común: cómic, tebeo e historieta. Según la RAE, cómic es una «serie o secuencia de viñetas que cuenta una historia» o «libro o revista que contiene cómics» (2001: 599); tebeo se define como una «publicación infantil o juvenil cuyo asunto se desarrolla en series de dibujos» o «serie de aventuras contada en forma de historietas gráficas» (2001: 2143) e historieta se considera una «fábula, cuento o relación breve de aventura o suceso de poca importancia» o «serie de dibujos que constituye un relato cómico, fantástico, de aventuras, etc., con texto o sin él, y que puede ser una simple tira en la prensa, una o varias páginas, o un libro» (2001: 1220). El término tebeo se utiliza sobre todo para los ejemplares completos, «historieta» designa «solo el medio y la obra como tal» (Castillo 
Cañellas 1996: 2), mientras la palabra «cómic» se usa en un sentido más generalizado y abarca tanto el arte de dibujar/contar como la completa producción de los cuadernos.

En la lengua francesa, la única palabra que designa este tipo de publicaciones es «bande dessinée» y en la lengua serbia «strip». A pesar de ser considerado literatura popular, literatura de masas o incluso paraliteratura, el cómic, surgido a finales del siglo XIX en los Estados Unidos, tiene dos elementos cruciales, como ya se ha dicho más arriba: el texto y la imagen, donde el contexto los hace inseparables.

\section{La traducción de los cómics}

Puesto que se trata de una de las ramas traductológicas todavía poco exploradas en las investigaciones académicas, luego de mencionar las características básicas acerca de la traducción de los cómics, nos enfocaremos en los problemas concretos de nuestro tema principal: la traducción de uno de los cómics mundialmente conocidos: el Astérix de René Goscinny y Albert Uderzo.

Para traducir tebeos, además de lo antes mencionado, un excelente conocimiento tanto del idioma de partida como de la lengua de destino y sus respectivas culturas, el traductor debe tener una gran creatividad y audacia. Según la afirmación de Silvia Palma (2006: 901), la traducción de los cómics se realiza en tres fases: comprensión desverbalización - re-expresión, lo que supone el entendimiento del sentido del texto, contexto y de la intención del autor, a propósito de transmitirlo al nuevo idioma. Uno de los dilemas cruciales en cuanto a la traducción de los cómics es su literal transmisión a otra lengua/ cultura o la adaptación a ella. De todas formas, «in any attempt to examine the communicative nature of the translating text, a number of assumptions will have to be made about texts, their users and the context in which they occur»4 (Hatim \& Mason 2014: 12). Además de eso: «jedna od nezaobilaznih karakteristika stripovnog stila jeste prisustvo ludičke funkcije jezika (funkcija igre, poigravanja jezikom)»5 (Maričić \& Popović 2014: 622) y asimismo «le traducteur doit donc prendre en considération les besoins, les connaissances préalables et les attentes du public cible et ne peut pas lui offrir la même quantité ni le même type d'informations que l'auteur du texte de départ»6 (Popović \& Nikolić 2017: 374).

4 «En cualquier intento de examinar la naturaleza comunicativa del texto traducido, se deberán hacer una serie de suposiciones sobre los textos, sus usuarios y el contexto en el que se producen.» (Traducido por las autoras de este artículo)

5 «Una de las características indispensables del estilo de tebeos es la presencia de la función lúdica de la lengua (la función de juego, jugar con la lengua).» (Trad. aut.)

6 «El traductor debe tener en cuenta las necesidades, el conocimiento previo y las expectativas del público objetivo y no puede ofrecer la misma cantidad o el mismo tipo de información que el autor del texto original.» (Trad. aut.)

BEOIBERÍSTICA Vol. IV / Número 1 (2020) | 77-94 
Un hecho comúnmente aceptado es que uno de los elementos más logrados de parte del autor y más difíciles de parte del traductor es el humor, en cualquier tipo de texto, ya que el humor es un fenómeno transcultural relacionado con la psicología y la sociedad de un pueblo, y se hace necesario tener en cuenta que él siempre está relacionado con el contexto. En otras palabras, la percepción del humor muchas veces no coincide sino varía dependiendo de los pueblos (Rollo 2017: 185). De hecho, «si hay un acto comunicativo difícil de traducir, y que por su complejidad merecería mayor análisis desde el punto de vista de la Pragmática, éste es el humor» (Campos-Pardillos 1992: 104). Traducir ese tipo de discurso exige producir el efecto humorístico del texto original en la audiencia de destino, lo que es todavía más difícil si la distancia entre las lenguas/culturas de origen y de destino es mayor, puesto que existe una estrecha conexión entre el texto y los aspectos socioculturales específicos de una comunidad (creencias, tradiciones, costumbres...).

En los cómics, el impacto del humor evidentemente está relacionado tanto con el texto como con la imagen. En cuanto a Astérix, hay que considerar que se trata de un personaje «muy» francés, con todos los matices de su cultura e historia. Por otro lado, su personaje «hace intervenir muchos estereotipos y particularismos locales o nacionales, que pueden pasar totalmente desapercibidos a los lectores que provienen de culturas muy alejadas (referencias culinarias, gastronómicas, musicales» (Palma 2006: 901), puesto que un lector común no tiene un conocimiento profundo sobre las características dialectales o referencias geográficas, sociales y culturales, en las cuales están basados los cuadernos de Astérix.

\section{Astérix y los cómics en Francia}

Cabe decir que los cómics típicos estadounidenses tienen una decena de páginas (publicadas en un papel de calidad baja) y las novelas gráficas unas doscientas (a todo color y edición lujosa); en Italia, el formato preferido es el de Sergio Bonelli Editore (cien páginas en blanco y negro) y en Francia en la mayoría de los casos se produce «the album, hard bound, large (A4) paper size, 48 to 64 pages and in full colours, sold in bookshops and addressed to an upper-market readership»7 (Zanettin 2014: 8).

El primer cuaderno, Astérix el Galo, que daría nombre a la serie, fue publicado con mucho éxito (300.000 copias vendidas) en el número inicial de la revista Pilote en 1959. Dos años después, el mismo cuaderno se publicó como cómic independiente en la Colección Pilote de la editorial Dargaud. A partir de entonces, se iban publicando nuevos

7 «El álbum, encuadernado, tamaño de papel grande (A4), de 48 a 64 páginas a todo color, vendido en librerías y dirigido a los lectores del mercado superior.» (Trad. aut.) 
álbumes, en tirajes cada vez más grandes. Por ahora hay 38 álbumes de Astérix, de los cuales 24 fueron firmados por René Goscinny y Albert Uderzo. Después de la muerte de Goscinny (en 1977), Uderzo continuó editando y publicando otros 10 álbumes. Los cuatro últimos tomos de la serie fueron firmados por Jean-Yves Ferry (guionista) y Didier Conrad (dibujante). Cabe mencionar que Astérix ha sido traducido a 107 lenguas y dialectos. Con más de 370.000.000 de ejemplares vendidos en 60 años en el mundo entero, se considera el cómic de mayor difusión del mundo.

\section{Astérix en las lenguas española y serbia}

Conviene resaltar que la primera traducción española de Asterix se publicó en 1965 y la serbo-croata en 1966 (Cros 2011: 20, 22). Asimismo, la versión serbia salió a la venta en 1995 (cinco años después de la desintegración de Yugoslavia). A pesar de que se han escrito varios artículos sobre Astérix, jamás se ha hecho una comparación de su versión francesa, española y serbia, por lo cual, para llevar a cabo esta investigación, no hemos podido tener en consideración artículos previos. Por consiguiente, nuestro corpus abarcó las traducciones hechas por Víctor Mora (al español) y Đorđe Dimitrijević (al serbio) de los siguientes cuadernos:

\begin{tabular}{|l|l|l|}
\multicolumn{2}{c}{ Título original } & \multicolumn{2}{c|}{ Versión francesa } & Asteriks među serbia \\
\hline Astérix chez les Belges & Astérix en Bélgica & Asteriks među Britancima \\
\hline Astérix chez les Bretons & Astérix en Bretaña & Asteriks u Švajcarskoj \\
\hline Astérix chez les Helvètes & Astérix en Helvecia & Asteriks u Španiji \\
\hline Astérix en Hispanie & Astérix en Hispania & Otmica Aspiriniksa \\
\hline Astérix et les Goths & Astérix y los godos & Asteriks i Normani \\
\hline Astérix et les Normands & Astérix y los normandos & Asteriks gladijator \\
\hline Astérix gladiateur & Astérix gladiador & Cezarov poklon \\
\hline Le cadeau de César & El regalo del César & Grad bogova \\
\hline Le domaine des dieux & La residencia de los dioses & \\
\hline Le tour de Gaule d'Astérix & La vuelta a la Galia por Astérix & Put oko sveta \\
\hline
\end{tabular}

Al comparar los títulos en los tres idiomas, podemos concluir que el traductor al español, para transmitir la preposición «chez», ha optado por la palabra «en» y en la sustitución del gentilicio con el nombre del país en tres casos: Astérix chez les Belges en su versión resulta Astérix en Bélgica y el mismo procedimiento aplica en Astérix chez les Bretons y Astérix chez les Helvètes, mientras el traductor serbio en las primeras dos ocasiones guarda la construcción francesa y en la tercera opta por sustituir el gentilicio con el nombre del país. Astérix en Hispanie en la traducción española transmite el mismo 
topónimo, pero en la versión serbia el traductor opta por la palabra «España», ignorando o pasando por alto la antigua nomenclatura de la Península Ibérica, designada por los romanos, de la cual iba a derivar el nombre actual. El título Astérix et les Goths sufrió la adaptación en la traducción serbia, resultando El secuestro de Aspirinix y el traductor serbio optó por la misma técnica en Le tour de Gaule d'Astérix, nombrándolo La vuelta al mundo. Por último, el cuaderno originalmente titulado Le domaine des dieux en la versión española se titula La residencia de los dioses y en la serbia $L a$ ciudad de los dioses.

De estos ejemplos podemos ver que solo cinco títulos coinciden en los tres idiomas, mientras que en otros cinco se aplica una de las técnicas de traducción, aplicada por los traductores respectivos.

\section{La traducción del contexto cultural: juegos verbales}

El contexto de Astérix por un lado facilita al traductor (la trama se desarrolla alrededor del año 45 a. C.; el protagonista siempre se mete en aventuras -en Galia o en los viajes por Europa- y los otros personajes son mayoritariamente masculinos), pero, por otro lado, exige de él un conocimiento profundo de las características de los pueblos y sus circunstancias histórico-sociales, para poder transmitir sus referencias, costumbres, símbolos, estereotipos. Sin embargo, los enfoques de nuestra investigación han sido las frases hechas, expresiones típicas y el humor verbal, presentes en varios contextos culturales. Los cuadernos de Astérix, cuya trama está ubicada en territorios y circunstancias socio-culturales específicos (reales o ficticios), abundan de referencias particulares y de juegos de palabras, casi siempre relacionados con personas y lugares. Aparte de los diálogos repletos de humor, en Astérix hay chistes sin palabras -el humor visual- que, por cierto, no hace falta traducir. Por otro lado, la onomatopeya, por ejemplo, no transmite solo un mensaje/contenido verbal, sino también visual.

Desde luego, «le texte source est créé pour correspondre à la situation dans la culture source. (...) Les langues et les cultures sont des entités différentes et que la valeur de chaque élément est définie par sa relation avec d'autres éléments du même système»8 (Popović \& Nikolić 2017: 359), por lo cual es necesario hacer varios cambios al transmitir el texto de un sistema linguiístico al otro. Según Meyer (2001), en la mayoría de los cuadernos de Astérix, el humor está basado en la parodia intercultural, el anacronismo de las relaciones internacionales, clichés geográficos y los juegos

8 «El texto origen fue creado para adaptarse a la situación en la cultura original. (...) Las lenguas y las culturas son entidades diferentes y el valor de cada elemento se determina en función de su relación con otros elementos del mismo sistema.» (Trad. aut.) 
interlingüísticos de palabras. Por ejemplo, Astérix en Hispania contiene una larga lista de juegos con topónimos, nombres propios y referencias culturales estereotípicas: Pompaleo (Pamplona), Helmantica (Salamanca), Corduba (Córdoba), Don Quijote y Sancho Panza, los molinos, la procesión de Semana Santa...

Los próximos ejemplos, extraídos de nuestro corpus, ilustran los problemas mencionados, con expresiones y frases conocidas o inventadas: "Je suis dans de beaux draps!» $(\mathrm{GL}, 29)$ / «iPobre de mí!» $(\mathrm{GLe}, 29)$ / «Sad sam obrao bostan!» (GLs, 28), donde cada frase corresponde con su propia cultura. Otro ejemplo lo demuestra todavía mejor:

Je ne sais pas, je voulais demander au Romain ce qu'il voulait frire dedans, mais il est tombé dans les pommes. $(\mathrm{AB}, 25)$

No sé, quería preguntarle al romano qué iba a freír, cuando el tipo se ha derrumbado como un saco de patatas. (ABe, 25)

Ne znam, ali izgleda da je sam sebi zapržio čorbu. (ABs, 24)

Por otro lado, en el siguiente ejemplo veremos que la versión original y la española contienen el mismo término cultural, siesta, pero el traductor serbio, a pesar de haber transcrito las palabras castellanas en otras ocasiones en el mismo tebeo, esta vez ha optado por la palabra serbia con el sentido adecuado, perdiendo el contexto cultural de la emblemática palabra española: «Aaaaah! Après ce bon repas, j’ai bien envie de faire une sieste!» (TG, 19) / «Ah, qué bien sienta una buena siesta después de semejante comida!» (TGe, 19) / «Aaah! Posle ovakve klope, dobro bi došla jedna dremka.» (TGs, 18).

\section{Particularidades lingüísticas}

Tanto las particularidades de las lenguas extranjeras habladas en los territorios que visita Astérix como las características de la lengua francesa utilizada en varios países francófonos, representan algunas de las fuentes de lo cómico. Por ejemplo, en el cuaderno titulado Astérix en Bélgica, el lenguaje cómico es principalmente el resultado de la intención del autor de imitar el idioma francés de los hablantes de Bélgica, y de las alusiones a los problemas culturales de ese país. Por consiguiente, habría que plantear la pregunta si los lectores de la versión española o serbia llegan a tener el conocimiento de las diferencias entre la lengua francesa utilizada en Francia y su variante belga, tal como lo tienen los lectores francófonos.

En el nivel léxico, Astérix en Bélgica abunda de palabras y expresiones típicas de ese país, denominadas como belgicismos; por ejemplo, la palabra carabistouille(s) $(\mathrm{AB}, 17)$ -el sustantivo cuyo sentido es broma, tontería, mentira- en la traducción española está reducida a la palabra broma, equivalente a la palabra serbia šala: «C'est pas la peine de raconter des carabistouilles» $(\mathrm{AB}, 17)$ / «Dejémonos de bromas» $(\mathrm{ABe}, 17)$ / «Šalu na 
stranu» (ABs, 16). El verbo déjeuner $(A B, 19)$, que en el francés estándar significa comer, ručati, en Bélgica se utiliza en el sentido desayunar, doručkovati. En las versiones española y serbia los traductores optaron por el sentido estándar, pero su decisión no influyó en el efecto cómico presente en el texto original. Hay más ejemplos cuando los dos traductores evitan transmitir las particularidades léxicas de la lengua francesa belga, como por ejemplo el sustantivo fieu $(\mathrm{AB}, 14)$, que siempre se utiliza en vocativo y su sentido es: mi amigo. El belgicismo drache -«Allons une fois nous mettre un peu à l'abri. On va attendre que cesse de tomber cette drache» $(\mathrm{AB}, 43)$ - que denomina un chubasco o aguacero, obtuvo diversos sentidos en respectivas traducciones: « $\mathrm{iVamos} a$ refugiarnos un poco! ¡Esperaremos a que cese esa llovizna!» (ABe, 43) / «Idemo malo u zaklon... Dok prestanu da biju ovim kamenčinama!» (ABs, 42), donde en la versión serbia el traductor ha optado por la palabra "piedras grandes, rocas" para aumentar la metáfora.

En el cuaderno La vuelta a la Galia por Astérix, Astérix y Obélix visitan varias regiones de la Francia actual. Los autores aprovechan ese episodio para ofrecer al lector las palabras y las expresiones regionales de la lengua francesa, e incluso varios acentos como por ejemplo el acento meridional de Marsella: «Môssieu» (a cambio de Monsieur) (TG, 31) / omitido en la versión española (TGe, 31) / Gospon (TGs, 30)- la versión abreviada del sustantivo gospodin (señor). Asimismo, para evocar el acento del sur de Francia, el traductor serbio a veces utiliza los elementos del habla montenegrina: «Ce sont eux!» (TG, 29) / «iSon ellos!» (TGe, 29) / «Bogomi su oni!» (TGs, 28), o en otra ocasión: «Oh, l’homme! Où que vous allez comme çà?» (TG, 30) / «iHola amigo! ¿Dónde vas tan resuelto?» (TGe, 30) / «A da, čoče, jadan ne bio, gde si se naputio?» (TGs, 29). La expresión «P'têt ben qu'oui. [...] P'têt ben qu'non.» (TG, 10), que se atribuye a los hablantes del francés de Normandía, significa probablemente y representa una variante regional de la expresión estándar: Peut-être bien qu'oui, peut-être bien que non. Su versión (más literal) en español es: «Puede que sí. [...] Puede que no...» (TGe, 10) y en serbio «Mož’ bit i tuda. [...] Mož’ bit i nije.» (TGs, 9), con el matiz regional.

Del cuaderno Astérix en Bretaña hemos seleccionado tres ejemplos donde el traductor serbio ha optado por expresiones falsas de la lengua inglesa, para producir el efecto cómico: «Splendide!» $(\mathrm{BR}, 8)$ / « ¿Espléndido!» $(\mathrm{BRe}, 8)$ / «Bjutiful!» (BRs, 7); luego «Merveilleux!» $(\mathrm{BR}, 10)$ / « Maravilloso!» $(\mathrm{BRe}, 10)$ / «Fajn!» (BRs, 9) y «Hello, patron!» $(\mathrm{BR}, 15)$ / «iHola, patrón!» $(\mathrm{BRe}, 15)$ / «Helou, gazda!» (BRs, 14). La misma técnica la ha aplicado en el cómic Astérix en Hispania, esta vez utilizando la palabra española, con el mismo motivo: «Ay, homme!» (AHI, 5) / « Vaya, hombre!» (AHIe, 5) / «Aj, hombre!» (AHIs, 4) o «Comment vous remercier, amis?» (AHI, 48) / «¿Cómo agradeceros esto, amigos?» (AHIe, 48) / «Kako da vam zahvalim, amigos?» (AHIs, 47). 
Obviamente, en estos ejemplos los traductores han utilizado diversas técnicas de traducción, que produjeron efectos más o menos logrados. Nuestra conclusión es que el traductor serbio en varias ocasiones ha optado por mayores modificaciones del texto regionalismos (bogomi, čoče), palabras extranjeras literalmente transcritas (bjutiful, fajn, helou, hombre, amigos) y varias expresiones relacionadas con la comida- para causar humor, y sobre todo para acercar las réplicas al lector serbio, mientras que el traductor español ha seguido la versión original con precisión en la mayoría de los casos analizados. Esto puede ser debido a la similitud del humor en la cultura española, no siendo así siempre en la cultura serbia.

\section{Frases hechas, dichos y refranes}

Para hacer una traducción lograda de un cómic exigente, como es el caso de Astérix, es necesario tener un profundo conocimiento del ámbito de su trama -una aldea ficticia ubicada al noroeste de Galia, rodeada de campamentos romanos- y sus características elementales -el humor basado en caricaturas, prejuicios y estereotipos, tanto de regiones francesas como de otras naciones europeas. Asimismo, es imprescindible conocer las circunstancias de la lengua meta, $y$, sobre todo, el sentido de humor de la cultura de destino. En otras palabras, no nos reímos de las mismas cosas y de las mismas circunstancias; la percepción del humor muchas veces no coincide sino varía dependiendo de los pueblos (Rollo 2017: 185).

Los siguientes ejemplos demuestran la habilidad de los autores de Astérix, pero también la creatividad de los traductores al español y al serbio. Cada frase encarna una tradición idiomática de las respectivas culturas y gente:

Tu es assez fort pour déraciner un arbre! (AN, 23)

¡Te sobra fuerza para arrancar un árbol de raíz! (ANe, 23)

Ti možeš volu rep da iščupaš! (ANs, 22)

Je n'ai jamais vu une mauvaise foi pareille ! $(A B, 19)$

¡Nunca he visto una mala fe como ésta! (ABe, 19)

Oho! Još nisam video da je neko tako pogan na jeziku! (ABs, 18)

Queremos destacar también otros ejemplos que demuestran la creatividad y la habilidad, sobre todo del traductor serbio, cuyas opciones se alejan ligeramente del original a propósito de acercarse al lenguaje coloquial y provocar el impacto cómico: «énerver» $(\mathrm{AN}, 24)$ / «ponerme nervioso» $(\mathrm{ANe}, 24)$ / «ići na ganglije» (ANs, 23); o en otra ocasión «se moquer» (AN, 30) / «burlarse» (ANe, 30) / «terati komendiju» (ANs, 29); y asimismo «tomber bien» (GL, 35) / omitido en la versión española (GLe, 35) / «doći kao kec na desetku»(GLs, 34). 
En los siguientes ejemplos podemos ver las equivalencias respectivas de cada lenguaje: «Ne te réjouis pas trop vite...» $(\mathrm{DD}, 16)$ / «No te alegres demasiado deprisa.» (DDe, 16) / «Prvo skoči, pa reci hop!» (DDs, 15); luego «Éno'mément de 'omains!» (BR, 5) / «iY son muchos romanos!» (BRe, 5) / «Ima ih ko žutih mrava!» (BRs, 4); o en otro cuaderno «Je n'ai pas eu de chance» $(\mathrm{CC}, 30)$ / «No he tenido suerte...» $(\mathrm{CCe}, 30) /$ «Bije me neki maler...» $(\mathrm{CCs}, 29)$ y «C'est qu'il y a un gros problème!» $(\mathrm{AH}, 41)$ / «iEs que hay un problema gordo!»(AHe, 41) / «U tom grmu leži zec!» (AHs, 40), donde el traductor serbio en el primer caso ha optado por un dicho popular, en el segundo ha usado el sintagma «hormigas amarillas» que se utiliza para designar una gran cantidad de algo/alguien, en el tercero ha transmitido el mensaje a través de una frase hecha popular y en el cuarto ha vuelto a optar por un dicho. A diferencia de la versión serbia, el traductor español ha transmitido de manera literal el texto francés, correctamente pero con menos creatividad.

\section{Expresiones coloquiales}

En los diez cuadernos de Astérix que forman parte de nuestro corpus, hay decenas de ejemplos de frases hechas, dichos y expresiones coloquiales que utilizan Astérix, Obélix y otros protagonistas. El ejemplo «Allons voir!» (DD, 19) tiene su equivalente en español: «iVamos a ver!» (DDe, 19), pero en la versión serbia el autor entra todavía más en la coloquialidad optando por la frase «Idemo da ždraknemo!» (DDs, 18), utilizando una expresión muy lograda y poco usada. En el ejemplo «À l'attaque!» (AN, 24) la traducción española corresponde con la francesa: «iAl ataque!» (ANe, 24), pero el traductor serbio modifica la frase añadiendo el matiz coloquial «Napreeeed! U makljažu!»(ANs, 23). Por otro lado, las expresiones «Il faudra le saisir avant qu'il s'envole!» (AN, 18) / « ¡Es precioso echarle mano antes de que se vaya volando!» (ANe, 18) / «Moramo ga drpiti pre nego što ispari!» (ANs, 17) tienen un valor verbal similar, dentro del marco coloquial.

En las próximas frases, el nombre personal Toutatis -dios galo de la guerra y los pueblos- ha sido transmitido a respectivas lenguas de maneras diferentes: en el primer caso, «Par Toutatis!» (TG, 7) / «Por Tutatis!» (TGe, 7) / «Svetog mu sveca!» (TGs, 6), el traductor español opta por la forma española de la misma palabra, Tutatis, mientras la traducción serbia contiene una expresión adaptada con la palabra «Santo», puesto que el lector serbio no habría entendido el mensaje si el traductor hubiera dejado «Toutatis». En otro cuaderno, los autores de Astérix vuelven a referirse al mismo dios: «Oh, et puis par Toutatis, assez!» (CC, 22), pero la versión española esta vez lo evita: «¡Oh, y basta de una vez!» $(\mathrm{CCe}, 22)$ y el traductor serbio escoge una lograda expresión coloquial «E, pa, sunce vam kalaisano, sad mi je dosta!» (CCs, 21). 
El análisis de estos ejemplos nos demuestra que los procedimientos del traductor español a veces están directamente relacionados con el hecho de que la lengua española y la lengua francesa tienen la misma raíz, por lo cual no es necesario utilizar tantas modificaciones de la traducción. Por otro lado, las opciones serbias revelan una gran creatividad del traductor y su excelente conocimiento de la lengua meta. Otros ejemplos que lo justifican se verán en la continuación de nuestro artículo.

\section{Adaptación cultural}

Cualquier tipo de traducción se realiza en base a la transferencia de un sistema cultural a otro, por lo cual es imprescindible conocer bien tanto las características de la cultura meta como la dimensión contextual del texto por traducir. Los elementos culturales «représentent des mots ou des combinaisons de mots typiques du mode de vie, de la culture, du développement social et culturel d'une société mais qui sont étrangers à d'autres sociétés, étant donné qu'ils désignent une spécificité locale ou historique»9 (Popović \& Nikolić 2017: 362).

La técnica titulada «la adaptación cultural» supone la sustitución de una palabra o expresión, típica de la cultura de origen, con otra palabra o expresión que no designa la misma realidad en la cultura meta, pero que pueda tener un impacto similar. Según M. Baker (1992: 31), la gran ventaja de esta estrategia de traducción es que ofrece al lector un concepto familiar y entendible. Para mostrarlo, hemos seleccionado de nuestro corpus los siguientes ejemplos: «Nous voudrions acheter une bouillabaisse pour emporter.» $(\mathrm{TG}, 31)$ / «Nosotros quisiéramos comprar una 'bouillabaisse' para recuerdo.» (TGe, 31) / «Tražimo girice da ponesemo.» (TGs, 30). Por cierto, la bouillabaisse es un plato tradicional de la Provenza francesa, una sopa de diversos pescados, que el traductor español ha prestado en su versión, pero que en la cultura serbia no tendría el efecto deseado, por lo cual el traductor optó por un término comúnmente conocido. Las mismas técnicas los traductores las han utilizado en el siguiente ejemplo: «Je vous offre le pastix?» (TG, 31) / «Les invito a un 'pastis'» (TGe, 31) / «Može jedna mastika?» (TGs, 30), puesto que el pastis (pastix en la versión original, porque los galos utilizan las palabras que terminan en -ix) es el aperitivo típico de Marsella y mastika es el licor tradicional macedonio, cuya referencia cultural es clara para los lectores serbios y se podría considerar un equivalente adecuado.

La adaptación cultural también es utilizada en las escenas cuando los protagonistas cantan, como en los siguientes ejemplos: «Je suis un petit garçon, fils de Gaulois moyen!» (AHI, 20), donde los autores hacen referencia a la canción «Petite fille

9 Representan palabras o combinaciones de palabras típicas en cuanto al estilo de vida, cultura, desarrollo social y cultural de una sociedad, pero ajenas a otras sociedades, ya que significan alguna especificidad local o histórica. (Trad. aut.)

BEOIBERÍSTICA Vol. IV / Número 1 (2020) | 77-94 
de Français moyen» de la cantante francesa Sheila, popular en los años 1970. Parece que la versión española no transmite la referencia adecuada o adaptada: «Anoche tuve un mal sueño, oh, Roma, dos triunviros me llevaban...» (AHIe, 20), mientras que la traducción serbia evoca los versos del gran poeta nacional Jovan Jovanović Zmaj: «Buji, paji, čedo moje, buji, paji...» (AHIs, 19), también aplicada en el siguiente ejemplo: «Dors mon p'tit Quinquin, mon p'tit Quinquin, mon p'tit Quintilius...» (TG, 16) -alusión en la homónima canción de cuna- / Duerme mi niño, duerme mi niño... (TGe, 16) / «Tiha noć je, moje zlato spava, nad glavom joj...» (TGs, 15).

Numerosos ejemplos de la adaptación cultural están relacionados con la comida, que producen un gran efecto cómico. Por cierto, en todas estas situaciones la sustitución es posible porque los platos emblemáticos no están dibujados / visualizados: "C'est qu'Idéfix et moi avons fini nos tartines.» $(A B, 33)$ / «Es que Idéfix y yo hemos acabado las rebanadas.» $(\mathrm{ABe}, 33)$ / «Znam, ali Garofiks i ja smo smazali sendviče!» (ABs, 32). En otra ocasión, el traductor español transmite la palabra francesa (puesto que se trata de una comida típica belga, obviamente reconocible en España) y el serbio opta por dos platos simples de desayuno -masa de avena y semolina de maíz: «Waterzooie! Waterzooie! Waterzooie! Morne plat!» $(\mathrm{AB}, 39)$ / «Este waterzooie no está muy bien hecho... El cocinero ha fracasado... ¡Qué derrota! ¡Es una derrota de waterzooie!» (ABe, 39) / «Ovsena kaša?! Ovsena kaša! Stradali su, kačamak im njihov!» (ABs, 38). Para aproximar las referencias culturales a los lectores en la lengua serbia, el traductor aplica la estrategia traslativa de domesticación a propósito de facilitar la comprensión del texto en el idioma objetivo, al mismo tiempo guardando las características emblemáticas de la versión original. Asimismo, introduce expresiones que abarcan las palabras (sobre todo las que designan comida) que evidentemente no existían en la época de la trama (kačamak, entre otras), pero que tienen un gran impacto humorístico. De esa manera, los lectores no tienen la impresión de la lectura de una traducción sino de la obra original, gracias a la creatividad y la fluidez del texto convincentemente transmitido.

Entre los casos más creativos se encuentran también los nombres propios, inventados por los autores franceses, con los equivalentes adecuados en las respectivas traducciones: «Soupalognon y Crouton»10 (AHI, 12) / «Sopalajo de Arrierez y Torrezno»11 (AHIe, 12) / «Supos de Crnolukos»12 (AHIs, 11), o Dansonsurlepon y Davignon (Dansons sur le pont y d'Avignon, que se refiere a la conocida canción tradicional francesa), en español designado Porrompompero y Fandánguez y en serbio Tangos de Slofoks.

10 Soupe à l'oignon - sopa de cebolla; croûtons - picatostes.

11 Sopa al ajo + ajoarriero (salsa de ajo), + torrezno (picatostes).

12 Sopa de cebolla, donde palabras "sopa" y "cebolla" están modificadas a lo español. 
Los últimos ejemplos de los elementos culturales que queremos destacar son asimismo los más delicados: en la primera escena de Astérix en Hispania, Pepe, el niño secuestrado por Julio César, se defiende de su invasión romana gritando «Vous ne passerez pas!» (AHI, 7), lo que obviamente hace referencia al lema republicano utilizado en la Guerra Civil española: «№ pasarán!». Desde luego, la frase fue traducida al español como «¡No pasaréis!» (AHIe, 7) -hay que tener en cuenta que este cuaderno se publicó en 1969, o sea durante el gobierno de Francisco Franco- y al serbio «Ovde nećete proći!» (AHIs, 6), aunque en ambos casos se hubiera podido aplicar la frase española: «No pasarán», puesto que el público lector tanto de España como de Serbia lo habría reconocido.

El último ejemplo que queremos mencionar en este artículo, relacionado con el ámbito histórico-cultural, tiene que ver con la tauromaquia. En una de las escenas del cómic Astérix en Hispania, llevada a cabo en la corrida de toros, los condenados «gladiadores» Astérix y Obélix deben luchar contra leones, pero en lugar de la fiera, aparece un toro. Empieza la lucha (la correra) acompañada por los gritos del público y, de repente, a una dama romana se le cae la capa roja en la arena. Cuando Astérix la recoge, el toro se vuelve «encaprichado por la capa» (AHI, 46) y en ese momento espontáneo, entre los gritos «Olé», Astérix evidentemente inventa e inaugura el espectáculo taurino. Conviene resaltar que en las tres versiones (francesa, española y serbia) está presente la interjección «Olé», con ligeros cambios gráficos: OLÉÉÉÉÉ! (AHI, 45); ¡OLÉ! ¡OLÉ! ¡OLÉ! (AHIe, 45); OLE! OLE! OLE! (AHIs, 44). Por tanto, estos ejemplos demuestran que la adaptación cultural no es necesaria cuando se trata de expresiones conocidas, reconocibles o globalmente aceptadas.

\section{Conclusiones}

Cada discurso, de cierta manera, refleja una cultura, o mejor dicho un sistema cultural. En el caso de la traducción, ese reflejo también abarca la dimensión contextual. En general, no hay traductores especializados para los cómics ni traductólogos dedicados exclusivamente a la teoría de ese tipo de traducción, pero existe cierto número de artículos académicos que lo investigan -en nuestra opinión todavía pocospuesto que se trata de un arte omnipresente, popular e influyente.

El fundamento de este análisis han sido las circunstancias socio-culturallingüísticas de un cómic globalmente conocido -Astérix-transmitidas a dos lenguas de diferente raíz: la española y la serbia. En cuanto a los elementos técnicos, hemos concluido que los cuadernos de Astérix en los tres idiomas -francés, español y serbiotienen el mismo formato y número de páginas. La calidad técnica de las tres versiones es similar, pero cuando se trata del contenido, los cuadernos traducidos son resultado de la 
interacción de una dimensión cultural y una dimensión lingüística particular de cada ámbito.

Comparando varias soluciones ofrecidas por los traductores Víctor Mora y Đorđe Dimitrijević en circunstancias contextuales específicas, se puede argumentar que el traductor español ha comunicado el mensaje de manera precisa y adecuada, mientras que el traductor serbio ha utilizado más técnicas traductológicas para lograrlo. La técnica prevaleciente en la versión serbia es la adaptación, puesto que su cultura es más lejana de la cultura original. Por otro lado, la función comunicativa y su papel respectivo están cumplidos en ambos idiomas. Nuestro análisis ha mostrado que la mayoría de las frases traducidas es acertada, el humor tiene más o menos el mismo efecto y el mensaje está propiamente transmitido en ambos idiomas meta. Por consiguiente, el hecho de que una lengua sea romance y otra eslava no tiene importancia crucial y no influye en el entendimiento del discurso, pero sí que contiene matices diferentes debido a la habilidad de los traductores.

Para aproximar las referencias culturales a los lectores en la lengua serbia, el traductor Dimitrijević ha aplicado en varias ocasiones la estrategia traslativa de domesticación a propósito de facilitar la comprensión del texto en el idioma meta, al mismo tiempo guardando las características emblemáticas de la versión original. Asimismo, hemos notado que el traductor serbio, aparte de adoptar una posición más activa que el traductor español, solía transmitir el mensaje entrando en todos los matices del contexto, alejándose más de la versión original para acercarla mejor a su público lector. Además, ha mostrado un gran conocimiento del léxico y discurso frecuentemente utilizado en los cómics, por lo cual hemos concluido que tiene más experiencia en esa rama de la traducción.

Podemos concluir que durante el procedimiento de traducción de los tebeos las pérdidas a veces son inevitables, especialmente cuando hay que transmitir las expresiones, los estereotipos o las referencias culturales de la versión original a otro idioma, debido a sus características particulares (lenguaje coloquial, particularidades lingüísticas, etc.). Aunque el traductor de los cómics se enfrenta a ciertos problemas cuando tiene que transmitir los elementos de una cultura a otra, se puede decir que muchas veces los soluciona debido al uso del humor adecuado, teniendo en cuenta las percepciones, competencias y conocimientos de los lectores de la cultura meta. 


\section{BIBLIOGRAFÍA}

Baker, Mona. In Other Words. A Coursebook on Translation. London: Routledge, 1992. Print.

Brandimonte, Giovanni. «La traducción de cómics: algunas reflexiones sobre el contenido lingüístico y no lingüístico en el proceso traductor». Metalinguaggi e metatesti. Lingua, letteratura e traduzione, XXIV Congresso AISPI (Padova, 23-26 maggio 2007). A. Cassol et al. (dir.). Roma: AISPI Edizioni, 2012. 151-168. Impreso.

Castillo Cañellas, Daniel. «El discurso de los tebeos y su traducción». Tebeosfera, 1996. Web. 15 Feb. 2020.

Cros, Bernard. «Du village d'Astérix au village global: historique de cinquante ans de succès». Le tour du monde d'Astérix. Bertrand Richet (éd.). Paris: Presses Sorbonne Nouvelle, 2011. 19-38. Imprimé.

Diccionario de la lengua española. Madrid: Real Academia Española, 2001. Impreso.

Groensteen, Thierry. Système de la bande dessinée. Paris: PUF, 1999. Imprimé.

Hatim, Basil, and Ian Mason. The Translator as Communicator. London and New York: Routledge, 2014. Print.

Maričić, Sanja, i Nataša Popović. «Učenje stranog jezika kroz strip: analiza i zastupljenost stripovne forme $u$ udžbenicima za francuski i španski kao strani jezik.» Jezici i kulture u vremenu i prostoru III (2014): 619-629. Štampano.

Meyer, Jean-Paul. «Quelques aspects du plurilinguisme dans Astérix et Lucky Luke». Creliana, hors-série, 1 (2001): 59-71. Imprimé.

Palma, Silvia. «La traducción de los elementos culturales: el caso de Astérix y Mafalda». La cultura del otro: español en Francia, francés en España. Manuel Bruña Cuevas et al. (coord.). Sevilla: Universidad de Sevilla, 2006. 900-909. Impreso.

Campos-Pardillos, Miguel Ángel. «Las dificultades de traducir el humor: Astérix le Gaulois - Asterix the Gaul - Asterix el Galo». Babel A.F.I.A.L.: Aspectos de filología inglesa y alemana 1 (1992): 103-123. Impreso.

Popović, Nataša, et Jelena Nikolić. «La traduction des références culturelles dans les bandes dessinées». Godišnjak Filozofskog fakulteta u Novom Sadu XLI.3 (2017): 357378. Imprimé.

Rodríguez Rodríguez, Francisco. Cómic y traducción: preliminar teórico-práctico de una disciplina. Madrid: Editorial Sindéresis, 2019. Impreso.

Rollo, Alessandra. "Aspects linguistiques et idéologico-culturels dans la traduction de l'humour. Le cas de la bande dessinée Agrippine». MonTI - Monografías de Traducción e Interpretación 9 (2017): 181-218. Imprimé.

Zanettin, Federico. Comics in Translation. London: New York: Routledge, 2014. Print.

\section{Corpus}


(AB) Goscinny, René, et Albert Uderzo. Astérix chez les Belges. Paris: Dargaud, 1984. Imprimé.

(ABe) -. Astérix en Bélgica. Barcelona: Grijalbo/Dargaud, 1992. Impreso.

(ABs) Gošini, Rene, i Alber Uderzo. Asteriks među Belgijancima. Novi Sad: Forum Marketprint, 1979. Štampano.

(AG) Goscinny, René, et Albert Uderzo. Astérix et les Goths. Paris: Dargaud, 1969. Imprimé.

(AGe) -. Astérix y los godos. Barcelona: Grijalbo/Dargaud, 1998. Impreso.

(AGs) «Otmica Aspiriniksa». Striputopija, 2014. Web. 11.04.2019.

(AH) Goscinny, René, et Albert Uderzo. Astérix chez les Helvètes. Paris: Dargaud, 1971. Imprimé.

(AHe) - Astérix en Helvecia. Barcelona: Grijalbo/Dargaud, 1998. Impreso.

(AHs) «Asteriks u Švajcarskoj». Striputopija, 2014. Web. 15.04.2019.

(AHI) Goscinny, René, et Albert Uderzo. Astérix en Hispanie. Paris: Dargaud, 1984. Imprimé.

(AHIe) - . Astérix en Hispania. Barcelona: Grijalbo/Dargaud, 1987. Impreso.

(AHIs) «Asteriks u Španiji». Striputopija, 2014. Web. 19.04.2019.

(AN) Goscinny, René, et Albert Uderzo. Astérix et les Normands. Paris: Dargaud, 1967. Imprimé.

(ANe) -. Astérix y los normandos. Barcelona: Grijalbo/Dargaud, 1998. Impreso.

(ANs) «Asteriks i Normani». Striputopija, 2014. Web. 18.04.2019.

(BR) Goscinny, René, et Albert Uderzo. Astérix chez les Bretons. Paris: Dargaud, 1997. Imprimé.

(BRe) -. Astérix en Bretaña. Barcelona: Grijalbo/Dargaud, 1998. Impreso.

(BRs) «Asteriks među Britancima». Striputopija, 2014. Web. 15.04.2019.

(CC) Goscinny, René, et Albert Uderzo. Le cadeau de César. Paris: Dargaud, 1988. Imprimé.

(CCe) -. El regalo del César. Barcelona: Grijalbo/Dargaud, 1998. Impreso.

(CCs) Gošini, Rene, i Alber Uderzo. Cezarov poklon. Novi Sad: Forum - Marketprint, 1987. Štampano.

(DD) Goscinny, René, et Albert Uderzo. Le domaine des dieux. Paris: Dargaud, 1971. Imprimé.

(DDe) - . La residencia de los dioses. Barcelona: Grijalbo/Dargaud, 1998. Impreso.

(DDs) Gošini, Rene, i Alber Uderzo. Grad bogova. Novi Sad: Forum - Marketprint, 1985. Štampano.

(GL) Goscinny, René, et Albert Uderzo. Astérix gladiateur. Paris: Dargaud, 1969. Imprimé.

(GLe) -. Astérix gladiador. Barcelona: Grijalbo/Dargaud, 1992. Impreso. 
(GLs) «Asteriks gladijator». Striputopija, 2014. Web. 16.04.2019.

(TG) Goscinny, René, et Albert Uderzo. Le tour de Gaule d'Astérix. Paris: Dargaud, 1969. Imprimé.

(TGe) -. La vuelta a la Galia por Astérix. Barcelona: Grijalbo/Dargaud, 1998. Impreso.

(TGs) «Put oko sveta». Striputopija, 2014. Web. 19.04.2019. 